\begin{tabular}{|c|c|}
\hline Title & Decreased electrodermal activity in patients with epilepsy \\
\hline Author(s) & Horinouchi, Toru; Sakurai, Kotaro; Munekata, Nagisa; Kurita, Tsugiko; Takeda, Y ouji; Kusumi, Ichiro \\
\hline Citation & $\begin{array}{l}\text { Epilepsy and behavior, 100, UNSP } 106517 \\
\text { https://doi.org/10.1016//.y ebeh.2019.106517 }\end{array}$ \\
\hline Issue Date & $2019-11$ \\
\hline Doc URL & http:/hdl .handle.net/2115/79653 \\
\hline Rights & $\begin{array}{l}\text { @ 2019. This manuscript version is made available under the CC-BY-NC-ND } 4.0 \text { license } \\
\text { http://creativecommons.org/icenses/by-nc-nd/4.0/ }\end{array}$ \\
\hline Rights(URL) & http://creativecommons.org/icenses/by-nc-nd/4.0/ \\
\hline Type & article (author version) \\
\hline File Information & Epilepsy Behav_100_106517.pdf \\
\hline
\end{tabular}

Instructions for use 


\section{Decreased electrodermal activity in patients with epilepsy}

2 Toru Horinouchi $^{{ }^{*}}$, Kotaro Sakurai ${ }^{1}$, Nagisa Munekata ${ }^{2}$, Tsugiko Kurita1 $^{1}$, Youji Takeda ${ }^{1}$,

3 Naoki Hashimoto ${ }^{1}$, Ichiro Kusumi ${ }^{1}$

$4{ }^{1}$ Department of Psychiatry, Hokkaido University Graduate School of Medicine, Sapporo, Japan

$5 \quad{ }^{2}$ Faculty of Computer Science and Engineering, Kyoto Sangyo University, Kyoto, Japan

7 1: [Postal address] 060-8638 west 5, north 14, north district, Sapporo city, Hokkaido, Japan

8 [Phone number] +81017065160

$9 \quad$ [Fax number $]+81017065161$

11 2: [Postal address] 603-8555 kamikamo honzan, kitaku, Kyoto city, Kyoto, Japan

12 [Phone number] +81 757051913

13 [Fax number] +81 757051438

$14 *$ Correspondence

15 Toru Horinouchi

16 Department of Psychiatry and Neurology, Hokkaido University Graduate School of Medicine

17 060-8638 west 5, north 14, north district, Sapporo city, Hokkaido, Japan

18 Phone number: +8101706 5160

19 Fax number: +8101706 5161 
20 Email address: tetsukawa1234@gmail.com

\section{$21 \quad$ Email Addresses}

22 Kotaro Sakurai: kootaroo@ab.auone-net.jp

23 Nagisa Munekata: nagisa.munekata@gmail.com

24 Tsugiko Kurita: tsugiko@med.hokudai.ac.jp

25 Youji Takeda: ytakeda02@gmail.com

26 Naoki Hashimoto: hashimona@gmail.com

27 Ichiro Kusumi: ikusumi@med.hokudai.ac.jp

28

29

30 


\section{Decreased electrodermal activity in epilepsy}

\section{Abstract}

32 Objective: Biofeedback therapy using electrodermal activity (EDA) is a new non-invasive therapy

33 for intractable epilepsy. However, the characteristics of EDA in patients with epilepsy are little

34 known; therefore, we assessed the EDA characteristics in patients with epilepsy.

35 Methods: A cross-sectional observational study was conducted in 22 patients with epilepsy and 24

36 healthy individuals. We collected information on demographic characteristics, EDA, and state

37 anxiety from both groups, and epilepsy diagnosis, seizure number per month, disease duration, and

38 number of anti-epileptic drugs (AED) from the epilepsy group. A wristband device was used to

39 measure resting EDA from both wrists for 10 minutes under controlled temperature and humidity.

40 We compared the EDA levels between the epilepsy group and the control group and examined

41 correlations between EDA and epilepsy-associated factors in the epilepsy group.

42 Results: A decreasing trend in EDA was observed during the first 1 minute from the start of the

43 measurement in 22 epilepsy patients (with or without seizures) compared with healthy controls ( $\mathrm{P}=$

44 0.12). However, a significant decrease in EDA was found in 18 epilepsy patients with seizures

45 compared with healthy controls $(-0.48$ versus -0.26 ; $\mathrm{P}=0.036)$. Furthermore, seizure frequency

46 showed a significant inverse correlation with EDA in the epilepsy group $(\rho=-0.50, P=0.016)$.

47 However, neither disease duration nor the number of drugs prescribed correlated with EDA in the

48 epilepsy group.

49 Significance: Marginally decreased EDA was observed in patients with epilepsy, and significantly

50 decreased EDA was found in patients with a higher seizure frequency. The present findings shed

51 light on the appropriateness of EDA-biofeedback therapy in epilepsy. 
53 Keywords: electrodermal activity, biofeedback therapy, seizure numbers, non-invasive,

54 galvanic skin response, intractable epilepsy

56 Abbreviations

57 BFT Biofeedback treatment

58 CNV Contingent negative variation

59 EDA electrodermal activity

60 SCP slow cortical potential

61

62 


\section{Decreased electrodermal activity in epilepsy}

\section{Original Research Articles}

\section{1. INTRODUCTION}

65 Epilepsy is a chronic disease triggered by excessive electric activity in cerebrocortical neurons that

66 causes repeated epileptic seizures, leading to a sudden loss of consciousness or convulsions. The

67 prevalence is roughly $1 \%$ worldwide $(1,2)$. Drug therapy suppresses seizures in about $70 \%$ of

68 patients; the remaining 30\% suffer from refractory epilepsy where seizures cannot be suppressed by

69 drugs. Surgical operation could be considered for refractory epilepsy. The seizure suppression rates

70 were high to some extent in resective surgery of the lesional focus (60-75\%), resective surgery of the

71 non-lesional focus (32-51\%) (3), and vagus nerve stimulation (8.0\%) (4). However, these surgical

72 treatments are highly invasive and burdensome on the patient; therefore, there is a great need for non-

73 invasive treatments.

74 Biofeedback treatment (BFT) aims to assist patients in drawing feedback from biological information

75 including heartbeat, respiration, and brainwaves using various techniques to enable them to adjust

76 these values voluntarily. BFT has been used for a variety of physical and mental disorders, including

77 migraine and attention deficit hyperactivity disorder $(5,6)$. Electroencephalographic BFT, which has

78 long been used for epilepsy treatment, has certain effects on epilepsy symptoms (7-9). Recently, BFT

79 for epilepsy using electrodermal activity (EDA-BFT), an index of peripheral sympathetic nerve

80 function, has been considered a promising non-invasive treatment (10-13).

81 EDA, which is the minute electrical activity measured on the skin surface, reflects peripheral

82 synthetic nerve function (14). More specifically, it represents changes in sweat gland activity

83 triggered by postganglionic cholinergic fiber activity in peripheral nerves (14). Because EDA shifts

84 acutely according to emotional changes, it has been used widely to measure emotional responses,

85 such as anxiety and fear, in the neuropsychological field $(15,16)$. Several studies have demonstrated 


\section{Decreased electrodermal activity in epilepsy}

86 that EDA is inversely correlated with cerebral cortex activity in patients with epilepsy $(17,18)$, and

87 the findings provide a basis for the hypothesis that increasing the level of EDA using BFT would

88 reduce cerebral cortex activity, thus resulting in seizure suppression.

89 Previous studies demonstrated an approximately 45\% decrease in epileptic seizures with the use of

90 EDA-BFT $(10,11)$, and seizure frequency decreased by more than $50 \%$ in 6 out of 10 individuals

91 (12). This rate of 50\% responders indicates that the therapeutic effects of EDA-BFT are comparable

92 with those of novel AED (14-60\%) (19, 20), vagus nerve stimulation (45\%) (21), and ketogenic diet

93 (53\%) (22). Although few findings are available regarding the long-term prognosis, one study

94 suggests that the seizure suppression effect can last for more than three years (23). Additionally,

95 EDA-BFT, which has been drawing attention as a stress management intervention in epilepsy, not

96 only reduces epileptic seizures but also improves psychiatric comorbidities, including major

97 depressive disorder and anxiety disorder (24).

98 The purpose of EDA-BFT is to increase the level of EDA in patients with epilepsy. However, little

99 has known about EDA characteristics in patients with epilepsy underlying BFT. Only one report

100 compared patients with epilepsy with healthy controls was available to the best of our knowledge,

101 which indicated that the EDA in patients with epilepsy might be increased (25). Moreover, it remains

102 unclear how epilepsy-related factors, such as seizure frequency, disease duration, and drug treatment,

103 affect EDA in patients with epilepsy. Clarifications of these issues would allow us to assess the

104 appropriateness of EDA-BFT. Therefore, this study compared EDA characteristics between epilepsy

105 patients and healthy controls and also investigated the relationship between epilepsy-related factors

106 and EDA characteristics in epilepsy patients. 


\section{Decreased electrodermal activity in epilepsy}

109 2.1 Study design

110 This was a single-center, cross-sectional, non-invasive controlled study conducted at Hokkaido

111 University Hospital, one of the epilepsy centers in Japan.

112 2.2 Standard protocol approvals, registrations, and patient consents

113 This study was approved by the institutional review board of Hokkaido University Hospital, and

114 written informed consent was obtained from all participants.

$115 \quad 2.3$ Study participants

116 Participants were recruited from outpatients with epilepsy who visited the Department of Psychiatry

117 at Hokkaido University Hospital from January 2016 to March 2018. Age- and sex-matched healthy

118 controls were also recruited.

119 Patients with more than 18 years of age and diagnosed with epilepsy according to International

120 League Against Epilepsy criteria were included in this study (26). Patients with hyperhidrosis or

121 hypohidrosis, which may directly affect EDA measurements; those with lesions or burns at

122 measurement sites; and those with concomitant mental disorders determined by the Diagnostic and

123 Statistical Manual of Mental Disorders 5 were excluded from this study.

124 The information on age, sex, and resting EDA was collected at the time of measurement, and anxiety

125 was assessed in both groups using state anxiety scores with the State and Trait Anxiety Inventory

126 (STAI) (27). Additionally, information on the epilepsy syndrome, seizure frequency, number of

127 prescribed AED, and disease duration was obtained from the epilepsy group. We defined "without

128 seizures” as no seizure for more than one year.

$129 \quad 2.4$ Measurement device and measurement environment 


\section{Decreased electrodermal activity in epilepsy}

130 An E4 wristband ${ }^{\circledR}$ (Empatica Inc., Milan, Italy), a wearable wristband device, was used for EDA

131 measurement. The E4 wristband, which adopts an external measurement technique using alternating

132 current, measures EDA with two dry silver-plated electrodes attached to the inner surface of the

133 wrist. The sampling rate is $4 \mathrm{~Hz}$, and the device is capable of measuring $0.01 \mu \mathrm{S}$ to $100 \mu \mathrm{S}$. Data

134 obtained from the E4 wristband are comparable with those obtained from the conventional, orthodox

135 EDA measurement technique in which wet electrodes are placed on the palm $(28,29)$. Therefore, the

136 E4 wristband has been used broadly in clinical studies (30, 31).

137 EDA measurement was carried out in a dark, quiet room with the participant sitting on a sofa. The

138 room temperature was set at $23{ }^{\circ} \mathrm{C}$, the humidity was set at $60 \%$, and brightness and ambient noise

139 were controlled (14). All measurements were conducted by the same investigator (TH) during the

140 same time frame (14:00-15:00).

1412.5 Measurement procedure

142 After entering the room, the participant was asked to sit on a sofa and fill out the STAI, an anxiety

143 assessment scale. Alcohol swab was used to clean the patient's wrists (14), and the patient was then

144 required to wear E4 wristbands on both wrists; noise-canceling headphones (QuietComfort 35

145 headphones $I \circledR$, Bose Corporation, Framingham, MA, USA) were used for the purpose of blocking

146 noise. The patient was instructed to not move his/her body while closing eyes, to feel relaxed, and to

147 not fall asleep. EDA measurement was started 1 minute after the instructions and continued for 10

148 minutes.

1492.6 primary and secondary outcomes 


\section{Decreased electrodermal activity in epilepsy}

150 The difference in resting EDA between the epilepsy group and the control group was determined as

151 the primary outcome. Correlations between resting EDA and seizure frequency, the number of drugs

152 prescribed, or disease duration were assessed as the secondary outcomes.

$153 \quad 2.7$ Statistical analysis

154 Individuals who fell asleep (14), were unable to remain still, or developed epileptic seizures during

155 measurement were excluded from the study. The t-test was used to compare resting EDA between the

156 epilepsy group and the control group. Spearman's rank method was used to examine correlations

157 between resting EDA and seizure frequency, the number of prescribed AED, or disease duration in

158 the epilepsy group. In addition, the t-test, $\chi 2$ test, and Wilcoxon signed-rank test were respectively

159 used to analyze age, sex, and state anxiety in these two groups. EDA data from the left and right

160 wrists were averaged for each participant in the analysis, and a log conversion was then performed to

161 obtain a normal distribution $(14,32,33)$. All P-values were two-tailed, and the significance level was

162 set at $\mathrm{P}<0.05$. R statistical software (version 3.3.3) was used for statistical analyses.

1632.8 Data availability statement

164 Anonymized data can be made available to qualified investigators upon request to the corresponding 165 author.

\section{3. RESULTS}

168 Twenty-two patients with epilepsy and twenty-four healthy individuals participated in this study

169 (Table 1). The measurements were carried out without problems, and no participants were excluded

170 from the analysis because of sleeping or epileptic seizures during measurement. The male-to-female 


\section{Decreased electrodermal activity in epilepsy}

171 ratio, age, or state anxiety did not significantly differ between the epilepsy group and the control

172 group. Among the 22 participants in the epilepsy group, 21 suffered from focal seizures, and 14 had

173 temporal lobe onset epilepsy. The average disease duration (22.5 years) was relatively long. All

174 participants in the epilepsy group used AED, and the average number of prescribed AED was 2.27.

175 Epileptic seizures were completely suppressed in four participants but were still observed in 18

176 participants in the epilepsy group. The seizure frequency varied greatly with an average frequency of

1778.4 per month and a maximum frequency of 40 per month.

178 The log-transformed average resting EDA during 10 minutes, the primary outcome, was -0.56 in the

179 epilepsy group and -0.50 in the control group, and no significant differences were observed between

180 these two groups (95\% CI, -0.08 to $0.21 ; \mathrm{P}=0.39)$. Participants in both groups showed gradually

181 declined EDA during the 10-minute duration, and the greatest difference between the two groups was

182 observed immediately after the start of the measurement (Fig. 1). Thus, the log-transformed average

183 EDA during 1 minute after the start of the measurement was then compared between the epilepsy

184 group and the control group. A trend of decreased EDA was observed in the epilepsy group

185 compared with the control group (-0.42 versus -0.26 ; $95 \% \mathrm{CI},-0.04$ to 0.36 ; $\mathrm{P}=0.12$ ). Subsequently,

18618 individuals in the epilepsy group, in whom epileptic seizures were still observed, were classified

187 as the epilepsy with seizures group, which was further compared with the control group. Notably, a

188 significantly decreased EDA was found in the epilepsy with seizures group compared with the

189 control group $(-0.48$ versus -0.26 ; $95 \% \mathrm{CI}, 0.02$ to $0.43 ; \mathrm{P}=0.04)$.

190 We further examined the secondary outcomes in the epilepsy group. A significant inverse correlation

191 was observed between the EDA during the first 1 minute from the start of measurement and seizure

192 frequency in the epilepsy group, and the correlation was moderate $(\mathrm{P}=0.02 ; \rho=-0.50)$ (Fig. 2). In

193 addition, other epilepsy-related factors, including the number of drugs prescribed and the disease 
194 duration, were not correlated with the EDA during the first 1 minute. Furthermore, no correlations

195 were observed between the EDA and state anxiety, age, or sex in all the groups.

\section{4. DISCUSSION}

198 4.1 Main results and their interpretations

199 This study demonstrated a decrease in EDA in patients with epilepsy and a greater decrease in

200 patients with a higher seizure frequency. During the first 1 minute from the start of measurement, the

201 EDA tended to be lower in the epilepsy group than in the control group and was significantly lower

202 in the epilepsy with seizures group than in the control group.

203 The EDA slowly decreased during the measurement duration in both the epilepsy and control groups,

204 and the observation could be explained by the physiological mechanism of EDA. EDA increases with

205 enhanced activity of the sympathetic nervous system during emotional stimuli and movements but

206 decreases with relaxation and rest (34). Hence, a series of behaviors, including entering the room,

207 sitting on the sofa, completing the STAI, wearing the E4 wristband and headphones, listening to the

208 instructions, and waiting for a minute until the measurement started, were reflected in the EDA at the

209 start of the measurement (35). However, when the patient remained at rest, sympathetic activity

210 started decreasing, and the EDA started decreasing accordingly.

211 The significant decrease in EDA was observed only in the first 1 minute from the start of

212 measurement in the epilepsy with seizures group compared with the control group. We speculate that

213 the series of behaviors before measurement could affect EDA; the patients with seizures were less

214 affected, while healthy controls were more affected. The greater decrease in EDA in the first 1

215 minute in epilepsy patients might be a consequence of reduced function due to repeated abnormal 


\section{Decreased electrodermal activity in epilepsy}

216 electrical activity in the central nervous system, which is responsible for generating EDA. The

217 limbic-hypothalamic system is known to constitute areas of the central nervous system responsible

218 for generating EDA (14). It has been demonstrated that stimulation of the amygdala (36) and

219 enhanced cognitive activity mediated by increased activity in the ventromedial prefrontal cortex

220 induce EDA (37). A decrease in EDA was previously demonstrated in patients with epilepsy who

221 underwent temporal lobectomy including that of the amygdala (38), indicating that defects in the

222 central nervous system induce a functional decrease. Moreover, individuals with a higher seizure

223 frequency tend to display a greater decrease in cognitive function $(39,40)$, and repeated abnormal

224 electrical activity damages the central nervous system function. Because 14 patients, accounting for

225 the largest portion in the epilepsy group in our study, had temporal lobe onset seizures, the greater

226 seizure frequency might be associated with the more severely impaired limbic system, thereby

227 leading to a decreased EDA.

228 A previous study by Drake et al. found transiently higher amplitude sympathetic skin responses (a type

229 of EDA) evoked by auditory or tactile stimuli in patients with epilepsy than in normal controls (25),

230 and the findings are inconsistent with our results that showed low EDA in epilepsy. Notably, their

231 study examined EDA changes in seconds just after the stimuli, while our study observed those in

232 minutes . Therefore, the different findings in these two studies cannot be compared directly.

233 Additionally, Drake et al. observed longer latency of sympathetic skin response after stimulation in

234 epilepsy patients than in normal controls, indicating that epilepsy patients have lower sympathetic

235 activity. Moreover, Lanteaume et al. demonstrated that epilepsy patients which have seizures evoked

236 by emotional stimuli were more vigilant toward threatening stimuli than those which do not have

237 seizures evoked by emotional stimuli. (41). To the best of our knowledge, no study has observed EDA

238 changes in both seconds and minutes after the stimuli, and no study has combined EDA and emotional

239 stimuli either; such studies might help understand the role of EDA in epilepsy. 


\section{Decreased electrodermal activity in epilepsy}

$241 \quad 4.2$ Autonomic nervous system

242 Many previous studies have investigated the role of the cardiac autonomic nervous system (ANS) in

243 sudden unexpected death in epilepsy. Ponnusamy et al. found that epilepsy patients showed increased

244 cardiac sympathetic activity and decreased parasympathetic activity during epileptic seizures (42),

245 and interictal discharges altered RR interval (43). However, a meta-analysis indicates that AED have

246 no significant effects on cardiac sympathetic or parasympathetic function (44), suggesting that AED

247 might not affect EDA. In fact, to our knowledge, no studies have demonstrated that AED could affect

248 EDA.

$249 \quad 4.3$ Confounders

250 Confounders affecting the resting EDA might not have significant effects on our study findings. The

251 impact of drugs is an important factor to be considered. In this present study, the number of AED did

252 not affect EDA. Other drugs such as those with a central noradrenaline inhibitory effect or an

253 anticholinergic effect are known to reduce the levels of EDA $(34,45)$. However, because no

254 participants in our study were taking those drugs, the impact of drugs was considered unrelated to our 255 study findings.

256 In addition, EDA is lower in older individuals than in younger individuals $(14,34)$ as well as in

257 males than in females $(14,34)$. However, these factors did not affect EDA in our study. Moreover,

258 EDA is known to increase in the dominant arm $(14,34)$. In this study, because EDA was measured

259 simultaneously from both wrists and the average was used for analysis, the arm dominance did not

260 affect our results. Further, African-Americans have been shown to have higher EDA than Caucasians 


\section{Decreased electrodermal activity in epilepsy}

261 (14); however, because all participants in this study were Japanese, racial differences are not needed

262 to be considered.

\subsection{Appropriateness of EDA-BFT in epilepsy}

264 EDA-BFT is considered an appropriate treatment. The principle behind the inhibitory effect of EDA-

265 BFT on epileptic seizures lies in the decreased excitability of the cerebral cortex due to increased

266 EDA. Accumulating studies have used the direct current component called the slow cortical potential

267 (SCP) as an index for excitation of the cerebral cortex. SCPs originate in the depolarization of

268 cortical pyramidal cells, which is caused by the input from the thalamus, and reflect excitation in a

269 broad range of cortical regions (46). Contingent negative variation (CNV), a type of SCPs, has been

270 found to be inversely correlated with EDA $(17,18)$. In fact, a decline in seizure frequency resulting

271 from EDA-BFT has been shown to be correlated with decreased shifts in CNV (17). Therefore,

272 EDA-BFT lowers excitation in the cortex by regulating the thalamocortical projection system. Our

273 study demonstrated a mild decrease in EDA in patients with epilepsy and a greater decrease in EDA

274 in patients with a higher seizure frequency. From this point, it is surmised that EDA-BFT, which can

275 increase EDA, would recover the decreased EDA closer to the normal level in epilepsy patients.

276 Thus, EDA-BFT, which lowers excitation of the cerebral cortex by increasing EDA, is a reasonable

277 treatment option.

\section{5 limitations of this study}

279 This study has several limitations. This study was designed to compare resting EDA; however,

280 differences in EDA between the epilepsy and control groups were observed only immediately after

281 the start of measurement, and EDA decreased to the same level in both groups during 10 minutes of

282 rest. Therefore, we used the EDA data obtained during the first 1 minute from the start of

283 measurement to reflect EDA in the waking state in daily lives. Because participants in both groups 


\section{Decreased electrodermal activity in epilepsy}

284 followed the same procedure before measurement, the present results are considered to represent the

285 difference in the properties of EDA between the epilepsy and control groups. However, it would be

286 better to measure EDA with stimulation tasks if the differences in EDA in daily lives between these

287 two groups should be assessed.

288 In addition, the number of participants in this study was small, with 22 individuals in the epilepsy

289 group and 24 individuals in the control group. Therefore, the small sample size might result in no

290 statistically significant differences between the epilepsy group and the control group, although a

291 decreasing trend in EDA was observed in the epilepsy group. Moreover, because the sample size was

292 small, epilepsy symptoms or seizure types were not assessed in these two groups.

293 In conclusion, this study demonstrated a decrease in EDA in patients with epilepsy and a greater

294 decrease in patients with a higher seizure frequency. EDA-BFT is a technique to increase EDA levels

295 in patients with epilepsy based on BFT. The present findings shed lights on the appropriateness of

296 EDA-BFT in suppressing epileptic seizures.

\section{ACKNOWLEDGMENTS}

299 The authors would like to express cordial gratitude to the Masaya Yamashita Lab at Asahi Kasei

300 Corporation for providing E4 wristbands and valuable comments. This work was supported by JSPS

301 KAKENHI (Grant Number, JP18K15505). The authors would like to thank Prof. Yoichi M. Ito at the

302 Department of Statistical Data Science at the Institute of Statistical Mathematics for his valuable

303 advice on the analytical methods. 
305 FUNDING

306 This work was supported by JSPS KAKENHI (Grant Number, JP18K15505).

307

308 CONFLICTS OF INTEREST

309 All the authors state that there is no direct conflict of interest.

311 AUTHOR CONTRIBUTIONS

312 T Horinouchi contributed to the design and conceptualization of the study and drafted the manuscript.

313 K Sakurai, N Munekata, and I Kusumi interpreted the data and revised the manuscript. T Kurita N

314 Hashimoto and Y Takeda revised the manuscript.

\section{ETHICAL PUBLICATION STATEMENT}

317 We confirm that we have read the Journal's position on issues involved in ethical publication and 318 affirm that this study is consistent with those guidelines.

321 The datasets generated for this study are available on request to the corresponding author. 


\section{Decreased electrodermal activity in epilepsy}

\section{REFERENCES}

324 1. Steer S, Pickrell WO, Kerr MP, Thomas RH. Epilepsy prevalence and socioeconomic

325 deprivation in England. Epilepsia 2014;55:1634-1641.

$326 \quad 2 . \quad$ Osakwe C, Otte WM, Alo C. Epilepsy prevalence, potential causes and social beliefs in

327 Ebonyi State and Benue State, Nigeria. Epilepsy Res 2014;108:316-326.

328 3. Téllez-Zenteno JF, Ronquillo LH, Moien-Afshari F, Wiebe S. Surgical outcomes in lesional

329 and non-lesional epilepsy: a systematic review and meta-analysis. Epilepsy Res 2010;89:310-318.

330 4. Englot DJ, Rolston JD, Wright CW, Hassnain KH, Chang EF. Rates and predictors of

331 seizure freedom with vagus nerve stimulation for intractable epilepsy. Neurosurgery 2016;79:345332353.

333 5. Cho SJ, Song TJ, Chu MK. Treatment update of chronic migraine. Curr Pain Headache Rep $334 \quad 2017 ; 21: 26$.

335 6. Duric NS, Assmus J, Gundersen D, Elgen IB. Neurofeedback for the treatment of children 336 and adolescents with ADHD: a randomized and controlled clinical trial using parental reports. BMC 337 Psychiatry 2012;12:1.

338 7. Sterman MB. Biofeedback in the treatment of epilepsy. Cleve Clin J Med 2010;77 Suppl 339 3:S60-S67.

340 8. Tan G, Thornby J, Hammond DC, Strehl U, Canady B, Arnemann K, et al. A meta analysis

341 of EEG biofeedback in treatment of epilepsy. Clin EEG Neurosci 2009;40:1-8.

342 9. Rockstroh B, Elbert T, Birbaumer N, Wolf P, Duching-Roth A, Reker M, et al. Cortical self343 regulation in patients with epilepsies. Epilepsy Res 1993;14:63-72. 


\section{Decreased electrodermal activity in epilepsy}

344 10. Nagai Y, Aram J, Koepp M, Lemieux L, Mula M, Critchley H et al. Epileptic seizures are

345 reduced by autonomic biofeedback therapy through enhancement of fronto-limbic connectivity: a

346 controlled trial and neuroimaging study. EBioMedicine 2017;27:112-122.

347 11. Kotwas I, McGonigal A, Khalfa S, Bastien-Toniazzo M, Bartolomei F, Micoulaud-Franchi

348 JA. A case-control study of skin conductance biofeedback on seizure frequency and emotion

349 regulation in drug-resistant temporal lobe epilepsy. Int J Psychophysiol 2018;123:103-110.

350 12. Nagai Y, Goldstein LH, Fenwick PBC, Trimble MR. Clinical efficacy of galvanic skin

351 response biofeedback training in reducing seizures in adult epilepsy: a preliminary randomized

352 controlled study. Epilepsy Behav 2004;5:216-223.

353 13. Kotwas I, Micoulaud-Franchi JA, Bartolomei F, Nagai Y. Commentary: Integrating

354 electrodermal biofeedback into pharmacologic treatment of grand mal seizures. Front Hum Neurosci $355 \quad$ 2015;9:1-3.

356 14. Wolfram B. Electrodermal activity, 2nd Ed. New York: Springer Science+Business Media,

357 LLC; 2012;36-336

358 15. van der Ploeg MM, Brosschot JF, Versluis A, Verkuil B. Peripheral physiological responses

359 to subliminally presented negative affective stimuli: a systematic review. Biol Psychol 2017;129:131-

360153.

361 16. Meyer B, Yuen KS, Ertl M, Plomac N, Mulert C, Buchel C, et al. Neural mechanisms of 362 placebo anxiolysis. J Neurosci 2015;35:7365-7373. 


\section{Decreased electrodermal activity in epilepsy}

363 17. Nagai Y, Critchley HD, Rothwell JC, Duncan JS, Trimble MR. Changes in cortical potential 364 associated with modulation of peripheral sympathetic activity in patients with epilepsy. Psychosom 365 Med 2009;71:84-92.

366 18. Nagai Y, Critchley HD, Featherstone E, Fenwick PBC, Trimble MR, Dolan RJ. Brain

367 activity relating to the contingent negative variation: an fMRI investigation. Neuroimage

$368 \quad 2004 ; 21: 1232-1241$.

369 19. Cramer JA, Ben Menachem E, French J. Review of treatment options for refractory

370 epilepsy: New medications and vagal nerve stimulation. Epilepsy Res 2001;47:17-25.

$37120 . \quad$ Beyenburg S, Stavem K, Schmidt D. Placebo-corrected efficacy of modern nonenzyme-

372 inducing AEDs for refractory focal epilepsy: systematic review and meta-analysis. Epilepsia

$373 \quad 2012 ; 53: 512-520$.

374 21. Englot DJ, Chang EF, Auguste KI. Vagus nerve stimulation for epilepsy: a meta-analysis of 375 efficacy and predictors of response. J Neurosurg 2011;115:1248-1255.

376 22. Liu H, Yang Y, Wang Y, Tang H, Zhang F, Zhang Y, et al. Ketogenic diet for treatment of 377 intractable epilepsy in adults: A meta-analysis of observational studies. Epilepsia Open 2018;3:9-17.

378 23. Nagai Y, Trimble MR. Long-term effects of electrodermal biofeedback training on seizure

379 control in patients with drug-resistant epilepsy: two case reports. Epilepsy Res 2014;108:149-152.

380 24. Kotwas I, Mcgonigal A, Bastien-Toniazzo M, Bartolomei F, Micoulaud-Franchi JA. Stress

381 regulation in drug-resistant epilepsy. Epilepsy Behav 2017;71:39-50.

382 25. Drake ME, Andrews JM, Castleberry CM. Electrophysiologic assessment of autonomic

383 function in epilepsy. Seizure 1998;7(2), 91-96. 
384 26. Fisher RS, Acevedo C, Arzimanoglou A, Bogacz A, Cross H, Elger CE, et al. ILAE official

385 report: a practical clinical definition of epilepsy. Epilepsia 2014;55:475-482.

386 27. Spielberger CD, Smith LH. Anxiety (drive), stress, and serial-position effects in serial387 verbal learning. J Exp Psychol 1966;72:589-595.

28. Ragot M, Martin N, Em S, Pallamin N. Emotion recognition using physiological signals:

389 laboratory vs. wearable sensors. In: Ahram T, Falcão C, editors. Advances in human factors in

390 wearable technologies and game Design. AHFE 2017; Advances in intelligent systems and

391 computing, vol 608. Cham: Springer; 2018:15-22.

392 29. Poh M-Z, Swenson N, Picard RW. A wearable sensor for unobtrusive, long-term assessment 393 of electrodermal activity. IEEE Trans Biomed Eng 2010;57:1243-1252.

394 30. Onton JA, Kang DY, Coleman TP. Visualization of whole-night sleep EEG from 2-channel 395 mobile recording device reveals distinct deep sleep stages with differential electrodermal activity. 396 Front Hum Neurosci 2016;10:1-12.

397 31. Corino VDA, Laureanti R, Ferranti L, Scarpini G, Lombardi F, Mainardi LT. Detection of 398 atrial fibrillation episodes using a wristband device. Physiol Meas 2017;38:787-799.

399 32. Clamor A, Hartmann MM, Köther U, Otte C, Moritz S, Lincoln TM. Altered autonomic 400 arousal in psychosis: an analysis of vulnerability and specificity. Schizophr Res 2014;154:73-78.

401 33. Venables PH, Christie MJ. Electrodermal Activity. In: Martin I, Venables PH, editors.

402 Techniques in psychophysiology. Chichester: John Wiley \& Sons, Ltd.; 1980;3-67.

403 34. Niimi Y, Suzuki J. Electrodermal Activity. Tokyo: Seiwa Shoten Publishers; 1986;19-209 
Decreased electrodermal activity in epilepsy

404 35. Lang H, Tuovinen T, Valleala P. Amygdaloid afterdischarge and galvanic skin response.

405 Electroencephalogr Clin Neurophysiol 1964;16:366-374.

406 36. Critchley H. Volitional control of autonomic arousal: a functional magnetic resonance study.

407 Neuroimage 2002;16:909-919.

408 37. Kubota Y, Sato W, Murai T, Toichi M, Ikeda A, Sengoku A. Emotional cognition without 409 awareness after unilateral temporal lobectomy in humans. J Neurosci 2000;20:RC97.

$410 \quad 38 . \quad$ Rayner G, Jackson GD, Wilson SJ. Mechanisms of memory impairment in epilepsy depend 411 on age at disease onset. Neurology 2016;87:1642-1649.

412 39. Lin H, Holmes GL, Kubie JL, Muller RU. Recurrent seizures induce a reversible impairment 413 in a spatial hidden goal task. Hippocampus 2009;19:817-827.

414 40. Lotufo PA, Valiengo L, Benseñor IM, Brounoni AR. A systematic review and meta-analysis 415 of heart rate variability in epilepsy and antiepileptic drugs. Epilepsia 2012;53:272-282.

416 41. Lanteaume L, Bartolomei F, Bastien-Toniazzo M. How do cognition, emotion, and 417 epileptogenesis meet? A study of emotional cognitive bias in temporal lobe epilepsy. Epilepsy Behav $418 \quad 2009 ; 15: 218-224$.

419 42. Ponnusamy A, Marques JLB, Reuber M. Comparison of heart rate variability parameters

420 during complex partial seizures and psychogenic nonepileptic seizures. Epilepsia 2012;53:13144211321.

422 43. Zaatreh MM, Quint SR, Tennison MB, D’Cruz O, Vaughn BB. Heart rate variability during 423 interictal epileptiform discharges. Epilepsy Res 2003;54:85-90. 
Decreased electrodermal activity in epilepsy

424 44. Hata T, Kita T, Yoneda R, Tanada S. Effects of exogenous stimuli and centrally acting drugs

425 on galvanic skin responses in rats. Japan J Pharmacol 1981;31:23-31.

426 45. Birbaumer N, Elbert T, Canavan AGM, Rockstroh B. Slow potentials of cerebral cortex and 427 behavior. Physiol Rev 1990;70:1-41.

428 46. Micoulaud-Franchi JA, Kotwas I, Lanteaume L, Berthet C, Bastien M, Vion-Dury J, et al.

429 Skin conductance biofeedback training in adults with drug-resistant temporal lobe epilepsy and

430 stress-triggered seizures: a proof-of-concept study. Epilepsy Behav 2014;41:244-250.

\section{TABLES}

432 Table 1. Background information of participants in the epilepsy and control groups

\begin{tabular}{|c|c|c|c|c|}
\hline & \multicolumn{2}{|c|}{ epilepsy } & \multirow[t]{2}{*}{ control } & \multirow[t]{2}{*}{ p-value } \\
\hline & $\begin{array}{c}\text { with/without } \\
\text { seizures }\end{array}$ & with seizures & & \\
\hline number & 22 & 18 & 24 & N/A \\
\hline age $\dagger$ & $40.3(20-64)$ & $40.7(20-64)$ & $40.4(29-60)$ & $P=0.68$ \\
\hline female $\ddagger$ & 15 & 12 & 14 & $\mathrm{P}=0.49$ \\
\hline state anxiety§ & $40.4(24-66)$ & $40.4(24-66)$ & $38.1(20-55)$ & $P=0.62$ \\
\hline diagnosis & FE 21, GE 1 & FE 18 & $\mathrm{~N} / \mathrm{A}$ & N/A \\
\hline
\end{tabular}


Decreased electrodermal activity in epilepsy

\begin{tabular}{|c|c|c|c|c|}
\hline diagnosis in FE & $\begin{array}{c}\text { TLE 14, FLE 5, } \\
\text { OLE 1, UK 1 }\end{array}$ & $\begin{array}{c}\text { TLE 13, FLE 3, } \\
\text { OLE 1, UK 1 }\end{array}$ & N/A & N/A \\
\hline $\begin{array}{c}\text { seizure number } \\
\text { (/month) }\end{array}$ & $8.4(0-40)$ & $10.3(0.3-40)$ & N/A & N/A \\
\hline disease duration \\
(year)
\end{tabular}

433 The number in parentheses of each item indicates the range. $\dagger$, t-test; $\ddagger, \chi^{2}$ test; $\S$, Wilcoxon signed-

434 rank test. FE, focal epilepsy; GE, generalized epilepsy; TLE, temporal lobe epilepsy; FLE, frontal

435 lobe epilepsy; OLE, occipital lobe epilepsy; UK, unknown; AED, anti-epileptic drug.

\section{FIGURE LEGENDS}

Figure 1. Measurement results for the resting electrodermal activity (EDA) in each group

439 The graph represents the resting EDA during the test duration in the epilepsy with/without seizures

440 group, the epilepsy with seizures group, and the control group. A decreasing trend in EDA during the

441 first 1 minute from the start of measurement was observed in the epilepsy with/without seizures

442 group (95\% CI, -0.04 to 0.36; P = 0.12), and a significant decrease in EDA was found in the epilepsy

443 with seizures group (95\% CI, 0.02 to $0.43 ; \mathrm{P}=0.04)$. 
Decreased electrodermal activity in epilepsy

445 Figure 2. A scatter plot of seizure frequency and EDA levels in the first 1 minute after the start

446 of measurement in the epilepsy group

447 The seizure frequency showed a significant inverse correlation with EDA levels $(\mathrm{P}=0.02 ; \rho=-0.50)$. 


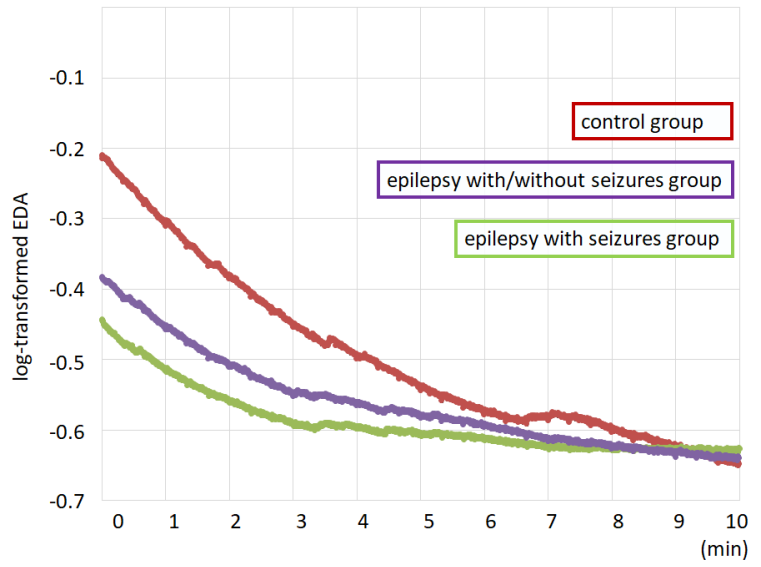




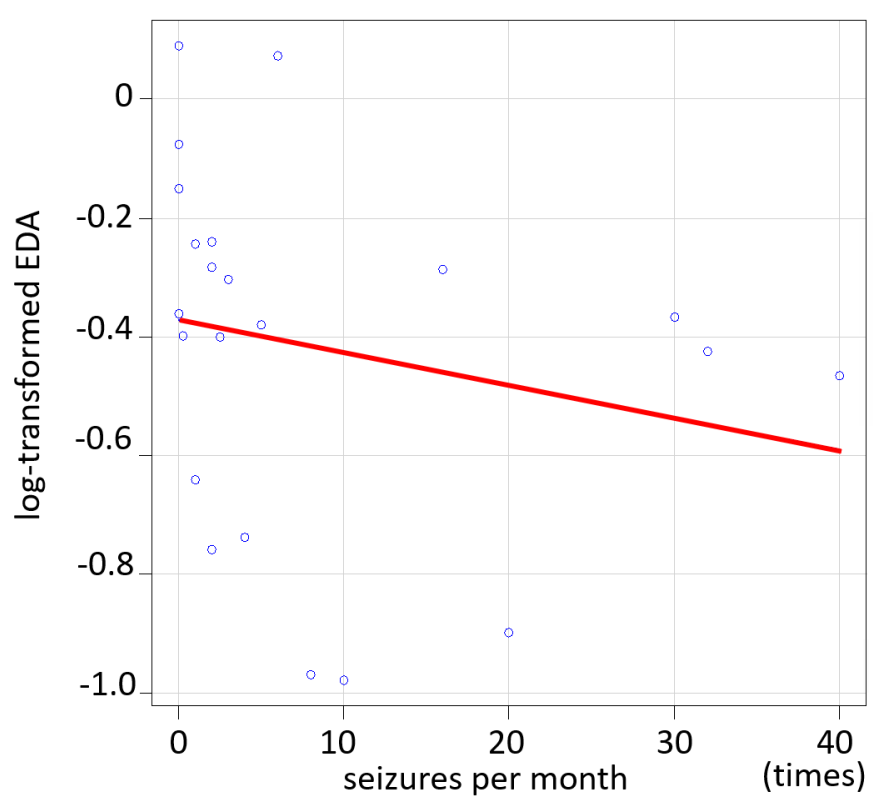

